\title{
A Collaborative Approach to Treatment of Alzheimer's Disease from a Psychological Perspective
}

\author{
Anna Maria Jadwiga Wegierek • Katarzyna Lesniak • Shan Patel • \\ Yuliya Bogdanovskaya \\ Wegierek Psychology Center, Wegierek Psychology Center, Inc, Chicago, IL, USA
}

Author for correspondence: Anna Maria Jadwiga Wegierek, Wegierek Psychology Center, Wegierek Psychology Center, Inc, 3022 N Harlem Ave, Suite 1N, Chicago, IL 60634, USA. Email: dr.wegierek@wegierekpsychology.com

Doi: http://dx.doi.org/10.15586/alzheimersdisease.2019.ch15

\begin{abstract}
After the age of 60, earlier in many cases, patients who experience perseverations, forgetfulness, and difficulties with daily living are often referred by their physicians for a neuropsychological evaluation. A neuropsychological evaluation consists of a variety of tests that illustrate a patient's cognitive functioning that include attention, concentration, verbal memory, visual memory, problem-solving, and cognitive flexibility. It further clarifies a range of diagnostic criteria that distinguish Alzheimer's disease (AD) from other mental healthrelated disorders. Depression presents in a very similar pattern to early stages of $\mathrm{AD}$. Therefore, the neuropsychological evaluation will rule in or out diagnostic criteria and pinpoint which medication should be recommended. A collaborative approach between psychologists, physicians, and caretakers is crucial in obtaining an accurate diagnosis to develop an appropriate treatment plan. Results from the neuropsychological assessment provide physicians with information to develop a medication regimen that helps treat a patient's cognitive and behavioral symptoms. Additionally, this information provides caretakers with psychoeducation to help understand the current functioning of their loved ones.
\end{abstract}

In: Alzheimer's Disease. Thomas Wisniewski (Editor), Codon Publications, Brisbane, Australia. ISBN: 978-0-646-80968-7; Doi: http://dx.doi.org/10.15586/alzheimersdisease.2019

Copyright: The Authors.

License: This open access article is licensed under Creative Commons Attribution 4.0 International (CC BY 4.0). https://creativecommons.org/licenses/by-nc/4.0/ 
The neuropsychological test findings coupled with a medical intervention is imperative to help patients and their families to develop adaptive methods that may help minimize the difficulties of daily living.

Keywords: Alzheimer's; attention; cognitive rehabilitation; dementia; emotional functioning; memory; neuropsychology

\section{INTRODUCTION}

Evaluating dementia, particularly Alzheimer's disease (AD), varies across settings. In this chapter, we will introduce what is neuropsychology, neuropsychological testing for possible or probable $\mathrm{AD}$, as well as the importance of collaboration between the doctors involved in treatment of the patient with $\mathrm{AD}$. In particular, we will describe what a medical doctor can expect from neuropsychological testing. Among countless batteries that are performed by neuropsychologists, we will emphasize the following phenomena: depression, substance use, decisionmaking, co-occurring mental health disorders, as well as the role that technology plays in neuropsychological testing. The importance of neuropsychological testing is priceless to patients. Results from neuropsychology testing can aid in confirming and/or ruling out often otherwise misdiagnosed syndromes that may require a different treatment plan and hence may impede and/or lead to success for appropriate treatment. For instance, neuropsychological testing may confirm $\mathrm{AD}$ and rule out depression and vice versa. Our hope is that readers will be more familiar with neuropsychological testing and its role in treatment for patients as well as their loved ones.

\section{WHAT IS NEUROPSYCHOLOGY?}

Neuropsychology is a scientific term that encompasses studying the central nervous system, including the brain and spinal cord, and how it influences an individual's cognitions and behaviors. This field within psychology has experienced a growth for over the last 40 years, with interest for about 120 years within the modern scientific psychology (1). Neuropsychology utilizes specialized knowledge of foundational neuroanatomy, principles of neuroscience, brain development, neurological disorders and etiologies, neurodiagnostic techniques, normal and abnormal brain functioning, and neuropsychological and behavioral manifestations of neurological disorders. In addition to this diverse amount of knowledge, neuropsychologists gather relevant historical information, conduct a neuropsychological examination, analyze and integrate data and findings, and provide feedback to the referral source. Neuropsychologists attempt to answer the question of "What mechanisms are responsible for human thinking, learning, and emotion, how do these mechanisms operate, and what are the effects of changes in brain states upon human behavior?" (1). This specialty applies principles of assessment and evidence-based interventions to the study of human behavior as it relates to normal and abnormal functioning of the central nervous system. The combination of extensive 
training, knowledge, and education with the process of obtaining and analyzing information allows for neuropsychologists to solve questions about the mind that otherwise cannot be solved.

As the main focus for neuropsychology is to determine the brain and behavior relationship with each patient exhibiting their own unique presentation, this has pushed to develop the field toward becoming an essential discipline for diagnosing and treating cognitive mental health disorders. Neuropsychology utilizes mechanisms to elicit activation in the multiple areas of the brain to determine their individual levels of functioning. In particular, these areas are referred to as cognitive domains. These cognitive domains studied by neuropsychologists include "perception, attention, learning, memory, processing of spoken and written language, thinking, reasoning, or belief formation, with the aim of learning more about the normal functional architectures of the cognitive processing systems used to carry out these activities" (2). As a result of testing the functioning in all the above-mentioned domains, there are a wide range of disorders that may be diagnosed including dementia, vascular disorders, Parkinson's disease, and other neurodegenerative disorders, traumatic brain injury, seizure disorders, learning disabilities (LD), neuropsychiatric disorders, infectious disease affecting the central nervous system (CNS), neurodevelopmental disorders, metabolic disease and neurological effects of medical disorders or treatment. Achieving a diagnosis allows individuals, their loved ones, their caretakers, and their physicians to gain awareness of the presenting problem and understand effective treatment recommendations to manage the diagnosis.

It is important to understand the direct correlation between cognitions and behaviors for neuropsychologists. This relationship that is observed is an individual's natural response to their environment, which may experience fluctuations. Neuropsychologist's main role is to attempt to distinguish where there are abnormalities and how it may cause dysfunction that may impact an individual's ability to properly function in their daily lives. Dysfunctions that a patient may experience may present in one or more of the domains of cognitive functioning. Each domain plays a crucial role in how individuals function in their personal and professional lives. These dysfunctions may present at different severities. In general, the minimal symptoms are not noticed by the patients; however, the more pronounced are usually noticed by their significant others. Many times, when a cognitive domain begins to decline, the patient may have minimal or no presenting symptoms, while others may have a severe presentation from the beginning. Such presentation depends on the domain that is impacted and possible other co-occurring medical complications. While there may be an overlap between the areas that present with similar problems, each has their own focus on how they contribute to the brain and cognition relationship.

One of the main impacts to the cognition and behavior relationship is a type of brain damage or trauma. The damage or trauma can range from a direct impact to the head to an organic occurrence in the brain. Regardless of the type of brain trauma, when it occurs, it can be localized or generalized throughout the brain. The area of where the impact occurs will influence the patient's presentation. It may be difficult to pinpoint where the impact occurred, but the neuropsychological evaluation will pinpoint impairments within each cognitive domain. The neuropsychological evaluation is pertinent as it will discern clusters of symptoms that may belong to psychiatric symptoms, medical conditions, as well as emotional presentation. 
The primary reason for a neuropsychological evaluation is to create a diagnosis and eliminate other disorders that may seem relevant but may not apply to the individual's presentation. Neuropsychological assessments specifically target parts of the brain through measuring the different cognitive domains of functioning. Cognitive functions are processes that help individuals carry out tasks requiring attention, memory, language, judgment, and problem solving. While it is well known that certain domains of cognitive functioning have their main centers, they also may be spread across several parts of the brain. Neuropsychological assessments elicit activity in different parts of the brain when activated with test tasks. A thorough analysis of the integrated data from the neuropsychological evaluation, will not only pinpoint given cognitive strengths but also weaknesses. Such analysis is most likely driven by patterns within the testing battery which as a result renders a diagnosis. This is why a neuropsychological evaluation contains a set of tests that measure similar cognitive functioning, rather than utilizing screening assessments. On that note, the neuropsychological evaluation is a lengthy process which oftentimes requires several hours of testing. While the several hours may seem long and daunting, it presents accurate data, rather than screening alone. Each neuropsychological battery is designed for an individual's ability to withstand long testing hours. In those situations a focused neuropsychological battery is conducted and a single cognitive domain may be tested (i.e., attention). Hence it is not uncommon that testing may vary in length from one to several hours, depending on the setting. For instance, testing may be shorter within an inpatient bedside setting and longer for a patient seeking an evaluation for a highprofile employment position, in an outpatient setting. In spite of the long hours of testing, it is our experience that patients and their family members appreciate the time that they engage in the testing, as this allows for a thorough evaluation. Patient's family members especially their children, appreciate the knowledge as they may carry similar genetic load and hence can utilize measures for prevention.

Individual results of the neuropsychological evaluation are compared to norms that will then indicate the level of functioning of this individual. The neuropsychological evaluation will render normative data that will indicate whether a patient is in the above average, average, or impaired range of functioning. The neuropsychological evaluation provides the clinician with twofold data. First, it delineates the pre-morbid functioning level that allows the neuropsychologist to understand whether the impairment is significant, as this may vary from one individual to another. For instance, if the tested individual has never experienced difficulties in school, it is expected that their premorbid functioning would demonstrate intact cognitions that are impaired during the time of testing. It is crucial to highlight here, the importance of having accurate norms to which the results are compared. The neuropsychological testing norms on several tests include norms not only for AD but also for patients with stroke, head trauma, LD, PTSD, and schizophrenia as compared to a control group. To further delineate neuropsychologists' work it is also important to note that each neuropsychological assessment presents not only an understanding of the cognitive domains in the human brain but also its characteristics that then determine diagnostic criteria and the appropriate treatment plan. 
For the purpose of diagnosing probable $\mathrm{AD}$, clinical neuropsychology is the main discipline to determine its presentation and severity. As aforementioned, AD impacts several areas of the brain and may present as several different diagnoses. It is imperative then to utilize the neuropsychological assessment results to determine an appropriate diagnosis and rule out other possibilities, as those treatment recommendations may be drastically different.

\section{NEUROPSYCHOLOGICAL TESTING FOR POSSIBLE OR PROBABLE AD}

$\mathrm{AD}$ is a neurodegenerative disorder. It is the most common type of dementia, with an estimate of 5 million individuals in the United States (65 or older) who were diagnosed with $\mathrm{AD}$ and related dementias in 2014 and an estimated 13.9 million projected to be diagnosed with the disease by the year 2060 (3). According to the World Health Organization, worldwide there are an estimated 47 million individuals affected by the disease currently (4). According to Braak and Braak (5), $\mathrm{AD}$ is "characterized by neuronal atrophy, synapse loss, and the abnormal accumulation of amyloidogenic plaques and neurofibrillary tangles in medial temporal lobe limbic structures (e.g., entorhinal cortex and hippocampus) and the association cortices of the frontal, temporal, and parietal lobes" (6). Several brain regions are affected by $\mathrm{AD}$ including the anterior cingulate cortex, the frontal lobes, as well as the medial temporal lobes (7).

According to Groth-Marnat (8), AD is referred to as a "cortical" dementia due to it affecting the cortical regions in the brain, as opposed to other types of dementia that affect the subcortical regions in the brain and cause impairments in attention and visuoconstruction. To further explain, the cortical dementias are characterized by more difficulties with memory and learning and sub-cortical effect more problems within attention and visuoconstruction.

The first cognitive symptom of $\mathrm{AD}$ is an impairment in episodic memory functioning, and later on as the disease progresses it impacts the frontal cortex, which impairs executive functioning (9). Based on a study conducted by Reed and colleagues (9), it was found that "Pathologically defined cases of Alzheimer's disease had verbal and non-verbal memory scores that averaged about 1 SD lower than their executive function scores." According to Smith and Bondi (10), the types of cognitive deficits observed in AD include impairments in areas such as memory and episodic learning, language and semantic knowledge, visuospatial, and executive functioning such as difficulties with problem-solving and abstract thinking as well as set-shifting. According to the Diagnostic and Statistical Manual of Mental Disorders (5 $5^{\text {th }}$ Edition), procedural memory and social cognition may be preserved for a longer period of time. Smith and Bondi further indicate that individuals with $\mathrm{AD}$ can experience anosognosia, in which they lack awareness of the cognitive problems they may experience (10). This lack of awareness may result in delayed diagnosis and treatment. Most patients are tested due to family concerns who notice the most salient characteristics such as perseveration and lack of new learning. 
Physicians may refer patients who experience forgetfulness, changes in their behavior and functioning, and/or a decline in the ability to complete activities of daily living independently, for a neuropsychological evaluation. When the referral question is for memory decline, it is important to conduct a thorough and detailed clinical interview. In such cases where the referred individual may have difficulties reporting relevant information and is deemed to be an unreliable historian, it is important when possible that the clinician attempts to obtain collateral information. This information may be obtained from the referred individual's caregiver or family member regarding the onset/frequency/severity of the individual's symptoms and functioning. Information obtained from the clinical interview guides the clinician to choose which test or series of tests need to be administered to the referred patient to address the referral question.

A standard neuropsychological testing battery to assess for possible or probable AD should include measures assessing several domains of functioning including: Attention, Processing Speed, Memory, Language, Executive Functioning and Dementia Severity (11). Simple attention continues to remain intact as patients with intact verbal skills, who present with severe impairments, are able to repeat 5 digits forward correctly (12). With regards to language abilities, individuals with AD often demonstrate impairments in verbal fluency, semantic fluency, and naming objects. Tests are administered to assess areas of visual and verbal memory, as well as the individual's performance in areas of immediate and delayed memory, and ability to learn new information. With regards to memory, patients with AD have difficulty encoding new information, the learning curve is flat across trials, and new information is not consolidated (13). Additionally, recognition is impaired and delayed recall of information is also poor, even after a short period of time (13). According to Delis et al. 1991, "...AD patients rapidly forget information over time and are equally impaired (relative to age-matched controls) on recognition and free recall components of the tasks. This pattern of performance is consistent with impaired consolidation rather than ineffective retrieval of new information" (14).

In addition to other criteria that must be met for a diagnosis of Major Neurocognitive Disorder Due to AD, the Diagnostic and Statistical Manual of Mental Disorders (5 ${ }^{\text {th }}$ Edition) indicates that there must be "clear evidence of decline in memory and learning and at least one other cognitive domain (based on detailed history or serial neuropsychological testing)" (15).

\section{DEPRESSION AND AD}

Neuropsychological tests help with determining if the patients have symptoms of dementia, depression, or both. As discussed above, depression and dementia share similar characteristics, but neuropsychologists know the differences. Neuropsychologists have the clinical capacity to rule out and state either diagnosis. Such luxury helps medical doctors and/or prescribing psychologists to design the most efficient treatment plan available for a patient.

There are many alternate diagnoses that may interfere with an accurate assessment of probable or possible AD. Depression is among one of the most common misdiagnoses due to the number of overlapping symptoms it shares with AD. 
The most common overlapping symptoms include loss of interest in enjoyable activities and hobbies, social withdrawal, problems with memory, lack of sleep or over-sleeping, and impaired concentration (16). The difficulty in differentiating AD from depression depends on several factors, which may include inaccurate information provided by the patient and/or family members, normal effects of aging, dementia and depression comorbid conditions (17). The causal relationship between dementia and depression is supported by findings that people with dementia appear to have a higher prevalence of depression (18). However, in many cases patients may not have $\mathrm{AD}$ or dementia and may just have depression. In such cases, particular attention is placed on depressive symptoms which are less common in dementia alone. These symptoms may include feelings of hopelessness, expressions of guilt, feelings of worthlessness, and thoughts of self-harm (19).

There have been correlations found between mild, moderate, and major depressive disorder and insomnia with regard to AD risk (20). Insomnia and depression share a complex relationship. Depression may cause sleep problems and sleep problems may cause or contribute to depressive disorders (21). However, in terms of diagnosing AD when lack of sleep and depression occurs, the challenge becomes greater, as the symptoms overlap. Some of the symptoms such as memory loss, poor judgment and taking longer to complete normal daily tasks, all may contribute to lack of sleep, depression, and/or AD itself. Again this is why a full neuropsychological evaluation is necessary as it encompasses evaluating sleep patterns, symptoms of depression, as well as highlights hallmarks of $\mathrm{AD}$. It is also crucial to state here that a neuropsychologist needs to work very closely with the patient's primary care physician, psychiatrist, and/or prescribing psychologist, as the medications that the patient is taking may also have a crucial impact on his/her functioning. "A survey in the United States of a representative sampling of 2206 community dwelling adults (age 62-85 years) was conducted by in-home interviews and use of medication logs between 2010 and 2011" (22). "At least 1 prescription medication was used by $87 \%, 5$ or more prescription medications were used by 36\%" (22). In summary, it is well known that an adult population that is most susceptible to AD takes about $1-5$ different medications a day. Those medications include not only medication related to systemic failures and/or inadequacies including steroids, diabetes medication, vascular problems, to list a few and they may cause side effects stimulating not only metabolic system problems, arrythmias, but also sleep problems.

In summary, a crucial part of the neuropsychological evaluation is evaluating sleep habits and to differentiate sleep difficulties related to emotional problems, side effects of medications, as well as poor sleep hygiene as they all require different interventions. Furthermore, Alhola and Polo-Kantolastate that attention, working memory, long term memory and decision making abilities can be impaired due to sleep deprivation (23). Moreover, metabolic functioning may also be impaired due to lack of sleep. While the body sleeps, a plumbing method called the glymphatic system opens up channels in the brain and allows fluid to flow rapidly throughout the brain, in order to flush out toxins or interstitial protein deposits (24) that may be accumulating in the brain (25). During sleep deprivation this accumulation of proteins or toxins may cause cognitive impairments, as our brain is unable to function at an optimal level. Although AD may not be the actual cause of these cognitive impairments for patients who lack 
sleep, the diagnosis of dementia becomes a viable conclusion. Most likely, this is due to the fact that when older individuals do not have quality sleep as well as struggle with other medical complications, they may simply develop functional impairment that may constitute dementia.

Anxiety can interfere with a person's everyday life and it is also the most common symptom throughout the different stages of dementia (26). Anxiety and agitation become more apparent in the early stages of $\mathrm{AD}$ as people begin to recognize their losses and the seriousness of the disease. They may become anxious about being left alone or abandoned, while any changes in the daily routine can also trigger anxiety and agitation (27). The prevalence of depression in AD is fairly high, with rates reported as high as $87 \%$ and averaging $30 \%$. Therefore, it is conceivable that comorbid anxiety and depression may affect a large percentage of patients with $\mathrm{AD}$ (28). The correlation is unknown between $\mathrm{AD}$ and anxiety; however, a recent study has shown that anxiety predicts risk of Alzheimer's disease ( $\mathrm{n}=26,193$ out of seven studies, hazard ratio $1.53,95 \%$ CI 1.16-2.01, $\mathrm{P}<0.01$ ) and vascular dementia $(\mathrm{n}=4916$ out of two studies, odds ratiol.88, 95\% CI 1.05-3.36, P < 0.01) (29). Although anxiety does not directly associate with the likelihood of having dementia or $\mathrm{AD}$, it may reflect neurodegeneration in patients who experience symptoms of AD or dementia.

\section{WHAT DO NEUROPSYCHOLOGISTS HAVE TO OFFER MEDICAL DOCTORS AND HOW IMPORTANT IS THEIR COLLABORATION}

Neuropsychologists are able to collaborate with primary care physicians and other specialty driven doctors in a variety of ways, including answering referral questions and establishing and clarifying diagnostic criteria related to dementia. Neuropsychologists use countless tests that are established to be a crucial part of dementia assessments in various situations. A few are described below.

The most common question with which patients, their family members, and their doctors come to neuropsychologists on an outpatient basis is regarding the gravity of dementia. In most cases, medical doctors complete the Mini Mental Status Exam (MMSE) to screen a patient's memory and attention. Typically, patients with dementia fail such questions. In other words, when patients are provided three words to learn and later recall, they do not remember the three words or they remember only one of the three words provided. In most situations, the patient that failed the memory and/or orientation tests are sent to a neurologist so an MRI and/or CT scan can be ordered to indicate the presence of any notable changes in the patient's brain scan. Sometimes, impaired patients may have normal MRIs or an MRI may show significant changes even while the patient's cognitive functioning seems intact. This is most likely explained by neuroplasticity. In such situations when the MRI and/or CT results do not correlate with the patient's self-report, neuropsychological testing is taken into consideration.

Another well-known issue establishing the need for completion of neuropsychological testing is the fact that patients remember previously learned tasks from the Mini-Mental Status Exam administered by their doctors. Repetition of similar 
tasks, for instance remembering three words, results in inconclusive data as the screening does not evaluate new learning but rather recall of previously learned information. Similar phenomenon is observed when adult children do not recognize symptoms of dementia in their parents. This happens due to repetition of similar conversations concerning daily tasks and/or activities that do not change.

Patients also tend to minimize their impairments when explaining them to their doctors, and their family members are unable to provide correct information about the patients' functioning because their loved ones have their routines. Usual rituals for family members often include calling the patient and asking them the same questions over and over. The questions they ask are most likely about the patient's eating habits and medication-taking rituals. Since going for a walk with the dog is not a novel task, the patients appear healthy and free from any cognitive problems. Neuropsychologists spend a lot of time with a patient's family, explaining to them that such routine is a very common phenomenon that promotes lack of accurate judgment about a family member's cognitive functioning. In fact, if the medical doctor and/or neuropsychologist hear any complaints relating to a family member's decreased cognitive functioning, such as perseverations and forgetfulness, the patient is most likely already in the advanced stages of dementia. Unfortunately, many patients reside alone and their beginning signs of dementia are not detected or are explained away as part of the normal aging process.

\section{THE ROLE OF ALCOHOL CONSUMPTION IN DEMENTIA}

Not all medical doctors choose to speak to their patients about their alcohol consumption. Most doctors that treat AD are younger than their patients. Both of these factors may often result in ambiguity and discomfort that may impede the doctor-patient dialog about alcohol use. However, most doctors of clinical psychology are well-equipped and clinically trained to discuss the topic of alcohol use with their patients.

Despite the toughness of this issue, it is well known that patients with $\mathrm{AD}$ are not immune to drinking alcohol. In fact, many dementia patients drink. Drinking causes falls, broken bones, and brain injuries. It also causes cognitive problems such as poor planning and organizational skills, poor decision-making and judgment, problems with impulsivity, difficulty controlling emotions, problems with attention and slower reasoning, lack of sensitivity to others' feelings, and behavior that is socially inappropriate. On the other hand, a systematic review by Peters and colleagues indicates that "in older people, small to moderate amounts of alcohol consumption are associated with reduced incidence of dementia and Alzheimer's disease (AD)" (30).

While there are more problems related to alcohol consumption, dementia and $\mathrm{AD}$ patients are getting contraindicated recommendations about drinking alcohol from their primary care physicians. In contrast, clinical psychologists with neuropsychology specialties are not only trained to treat alcohol use but are also able to perform neuropsychological tests that can characterize the type and extent of a patient's cognitive deficit as well as denote which symptoms belong to AD and which ones to alcohol use. In this way, rehabilitation efforts are maximized. 
The importance of neuropsychological testing in matters of AD and alcohol consumption was delineated by Robert Heirene, Bev John, and Gereth Roderique-Davies. The authors reviewed the available data and agreed that cognitive screening is limited and is trumped by far by comprehensive neuropsychological testing following screening to ensure a correct diagnosis (31). The authors stated that neuropsychological testing establishes crucial characteristics of alcohol related cognitive decline that mostly includes a decline in executive functioning and episodic, autobiographical, procedural, and working memory. They also discussed the issue of confabulation that may be related to both dementia itself and an alcohol related impairment (30).

\section{AD AND DECISION-MAKING CAPACITY}

Another type of neuropsychological referral encompasses denoting the gravity of $\mathrm{AD}$ symptoms, as well as the patient's decision-making capacity. Indeed, the vast majority of outpatient referrals relate to the individual who resides at home, and his/her family members worrying about their cognitive functioning. Patients with detected and undetected AD spend time alone at home. During those moments, they are vulnerable to burglary, deceit, and even death because their judgment is impaired. For instance, there have been individuals who opened the door to a stranger, soliciting them to withdraw money from the bank; as well as patients selling health insurance, buying homes, and even moving to another state without having a previous discussion or agreement with the rest of their family members about their plans. This demonstrates the lack of judgment, rational thinking, and insight which may be characteristic of individuals with dementias.

Another very important aspect for the need to complete neuropsychological testing regarding decisional capacity is the fact that many AD patients are in the hospital and are unable to make decisions regarding their health. Unfortunately, without completion of neuropsychological tests, many AD patients seem to cognitively function well to not only their family members but also to their doctors. Those are the patients that refuse certain treatments that may be very vital to their life. Those are the patients that lie in their hospital beds, seem unconcerned about their health, and lack an understanding about the gravity of their illnesses. Those are the patients that have shallow humor and tend to answer their doctors' questions with short answers that indicate their disinterest. For example, they tend to state that they do not care for politics hence they do not know who is the current president, and they tend to fail orientation tests even when the correct date was written by a nurse in front of them.

Families of AD patients have the tremendous task of accepting that their parents, uncles, aunts, and other family members who used to be sharp, have lost the ability to make decisions. The family members also vicariously deal with their own stress responses, facing the possibility that they may have similar genetic loads and that they may also develop AD. All of the above mentioned constraints lead to missing the symptoms, which then leads to the most dangerous problem: not doing anything.

After completion of the neuropsychological testing, the report and recommendations are delineated. One of the possible recommendations may be cognitive rehabilitation. Another recommendation may encompass an evaluation for a 
cognitive enhancer. Recommendations may also include possible counseling/psychotherapy and or a support group for the family members/caregivers to process their fears, help them develop skills to deal with their family member that has AD, and start the process of establishing a power of attorney.

\section{AD AND OTHER MENTAL HEALTH PROBLEMS}

Doctors of psychiatry often refer their patients for neuropsychological evaluations to rule out AD and possibly diagnose depression. They also seek neuropsychological evaluations to determine possible attention problems and undiagnosed LD that can all resemble early dementia.

It is our experience that more than a few referrals from psychiatrists include ruling out stress in patients as young as 50 years old that presented with dementia like symptoms. Those patients seem to have dementia, but neuropsychological evaluations are able to detect severe stress that results in their poor job performance. In such situations a conclusive diagnosis is crucial, as the treatment for stress as well as burnout differs from treatment for dementia.

Neuropsychological evaluations can also detect LD that have not previously been diagnosed. Nowadays, with more and more jobs requiring computer skills, this phenomenon occurs, even with those jobs that are considered computer-free. For instance, a 55-year-old janitor is now required to login to the computer to check memos, request days off, or perform required training. In this situation, a LD may mirror early dementia and hence lead to inappropriate treatment. Again, a neuropsychologist will not only test this individual's cognitive functioning but also recommend training, teach compensation skills, etc.

\section{AD AND TECHNOLOGY}

Typically, the older population does not do well with computerized testing, even though these kinds of tests are well-prepared for inexperienced computer users. Since computers were first used at schools in the eighties in the USA, patients that have never used a computer to study or work are included in this population. Hence, most patients that are at least 50 years old who lack the required computer skills, may be considered in this group. At our clinic, any time a computerized test was administered to an older population member, it has been observed that the cognitive abilities tested on the computer were lower than on paper and pencil tests. Hence, doctors using computer screening tests for cognitions may obtain inaccurate results. When in doubt about a patient's results, it is recommended that the patient be referred for a full neuropsychological testing battery.

\section{AD AND MARIJUANA USE}

Much of the older population in the United States recreationally consumes and/ or smokes marijuana, as it is now prescribed as well as highly available. When asked what their purpose for smoking is, most people state that it is healthy and 
that it helps them with sleeping and managing their pain. Research on smoking marijuana and its effects on cognitions vary, but in our practice it has been observed on several occasions, that severe delirium followed ingestion of cannabis. Cannabis use can result with hallucinations, as it is a hallucinogenic agent. It may also quicken cognitive decline, especially memory problems. While this has little to do with neuropsychological testing and $A D$, it is crucial to state here that all healthcare providers should consider referring any, but especially older patients, to neuropsychologists because the underlying causes of marijuana ingestion can be detected, processed, and potentially diminished; all of which would better their patient's quality of life. In other words, it is our belief that if an individual decides to smoke marijuana, he or she has reasons to do so. Those reasons may be depression, anxiety, withdrawal, loneliness, or many other problems. No matter the reason, in the therapeutic room of a skilled psychologist, those problems can be addressed, diminished, and/or possibly resolved.

\section{AD AND THE N-648}

The N-648 is a form that is used by medical doctors and psychologists to help immigrants obtain United States citizenship without passing the English proficiency and United States history exams. We believe that this form cannot be completed without normative data concerning the patient's memory. It is the neuropsychological testing that can actually determine whether an individual with probable or possible AD is capable of learning English and United States history, which are required to become a United States citizen.

\section{CONCLUSION}

Neuropsychological testing is underutilized and it is our hope that its use will be prevalent and treated as a necessity especially in patients with AD. Time spent on neuropsychological testing can change the patient's life tremendously. For instance, a depressed patient that actually has dementia after a neuropsychological assessment will not be prescribed anti-depressants. Conversely, a patient with dementia will be prescribed cognitive enhancers rather than anti-depressants. As mentioned above, testing may last from an hour to several hours, but the knowledge after it is completed is priceless and appreciated by the families we have served thus far. The neuropsychological testing not only helps the patient but also their families. We have spent countless hours working with our patients' families to not only help them with appropriate care, but also with the fact that they may be facing similar future presenting concerns. Those family members that worked with us had a tremendous advantage over their parents, as their anticipation and the possibility to carry similar genetic load allowed them to plan for the future and to initiate anything they can to avoid and/or delay AD. The neuropsychological reports written in our clinic are not only for the patients and their doctors, but also caregivers who can better understand the level of 
impairment, as well as learn skills to be a better support system. In other words, a few hours of neuropsychological testing is worthwhile for everyone that is involved with the AD patient.

Conflict of interest: The authors declare no potential conflicts of interest with respect to research, authorship, and/or publication of this chapter.

Copyright and Permission Statement: To the best of our knowledge, the materials included in this chapter do not violate copyright laws. All original sources have been appropriately acknowledged and/or referenced. Where relevant, appropriate permissions have been obtained from the original copyright holder(s).

\section{REFERENCES}

1. Graham Beaumont J. Introduction to neuropsychology. 2nd ed. New York: The Guilford Press; 2008. $382 \mathrm{p}$.

2. Coltheart M. Cognitive neuropsychology. Scholarpedia. 2008 Feb;3(2):3644. http://dx.doi. org/10.4249/scholarpedia.3644

3. Matthews KA, Xu W, Gaglioti AH, Holt JK, Croft JK, Mack D, et al. Racial and ethnic estimates of Alzheimer's disease and related dementias in the United States (2015-2060) in adults aged > 65 years. Alzheimers Dement. 2019 Jan;15(1):17-24. http://dx.doi.org/10.1016/j.jalz.2018.06.3063

4. World Health Organization. 10 facts on dementia [Internet]. Geneva: World Health Organization; 2017 Apr [cited 2019 Jul 1]. Available from: https://www.who.int/features/factfiles/dementia/en/

5. Braak H, Braak E. Neuropathological staging of Alzheimer-related changes. Acta Neuropathol. 1991 Sept; 82(4):239-59. http://dx.doi.org/10.1007/BF00308809

6. Salmon DP, Bondi MW. Neuropsychological assessment of dementia. Annu Rev Psychol. 2009 Jan;60:257-82. In: Albert MS, Moss MB, Tanzi R, Jones K. Preclinical prediction of AD using neuropsychological tests. J Int Neuropsychol Soc. 2001 Jul;7(5):631-9. http://dx.doi.org/10.1017/ S1355617701755105

7. Albert MS, Moss MB, Tanzi R, Jones K. Preclinical prediction of AD using neuropsychological tests. J Int Neuropsychol Soc. 2001 Jul;7(5):631-9. http://dx.doi.org/10.1017/S1355617701755105

8. Groth-Marnat G. Handbook of psychological assessment. 5th ed. Hoboken NJ: Wiley; 2009. 517 p.

9. Reed BR, Mungas DM, Kramer JH, Ellis W, Vinters HV, Zarow C, et al. Profiles of neuropsychological impairment in autopsy-defined Alzheimer's disease and cerebrovascular disease. Brain. 2007 Mar;130(Pt 3):731-9. http://dx.doi.org/10.1093/brain/awl385

10. Smith GE, Bondi MW. In: Morgan JE, Ricker JH, editors. Chapter 39: Normal Aging, mild cognitive impairment, and Alzheimer; Disease: Textbook of clinical neuropsychology. 1st ed. New York: Taylor \& Francis; c2008. p.762-780.

11. Weintraub S, Salmon D, Mercaldo N, Ferris S, Graff-Radford N, Chui H, et al. The Alzheimer's Disease Centers' Uniform Data Set (UDS): The neuropsychologic test battery. Alzheimer Dis Assoc Disord. 2009 Apr;23(2):91-101. http://dx.doi.org/10.1097/WAD.0b013e31819lc7dd

12. Deutsch Lezak M, Howieson DB, Bigler ED, Tranel D. Neuropsychological assessment. 5th ed. New York: Oxford University Press; 2012. 1200 p.

13. Zec RF. Neuropsychological functioning in Alzheimer's disease. In: Park RW, Zec RF, Wilson RS, editors. Neuropsychology of Alzheimer's disease and other dementias. 1st ed. New York: Oxford University Press; 1993. p. 3-80.

14. Delis DC, Massman PJ, Butters N, Salmon DP, Cermak LS, Kramer JH. Profiles of demented and amnesic patients on the California Verbal Learning Test: Implications for the assessment of memory disorders. Psychol Assess. 1991;3(1):19-26. http://dx.doi.org/10.1037/1040-3590.3.1.19

15. American Psychiatric Association. Dilip V. Joeste and Dan G. Blazer. Diagnostic and statistical manual of mental disorders. Arlington, VA: American Psychiatric Association; 2017. 611 p. 
16. Mayo Clinic. Alzheimer's or depression: Could it be both? [Internet]. Rochester: Mayo Clinic; [cited Jul 1]. Available from: https://www.mayoclinic.org/diseases-conditions/alzheimers-disease/in-depth/ alzheimers/art-20048362

17. Rotomskis A, Margevičiūtė R, Germanavičius A, Kaubrys G, Budrys V, Bagdonas A. Differential diagnosis of depression and Alzheimer's disease with the Addenbrooke's Cognitive Examination- Revised (ACE-R). BMC Neurol. 2014 Apr;15:57. http://dx.doi.org/10.1186/s12883-015-0315-3

18. Lyketsos CG, Steele C, Baker L, Galik E, Kopunek S, Steinberg M, et al. Major and minor depression in Alzheimer's disease: Prevalence and impact. J Neuropsychiatry Clin Neursci. 1997 Fall;9(4):556-61. http://dx.doi.org/10.1176/jnp.9.4.556

19. Thorpe L. Depression vs. dementia: How do we assess? Can Rev Alzheimers Other Dementias. 2009 Sept;12(3):17-21.

20. Pistacchi M, Gioulis M, Contin F, Sanson F, Marsala SZ. Sleep disturbance and cognitive disorder: Epidemiological analysis in a cohort of 263 patients. Neurol Sci. 2014 Dec;35(12):1955-62. http:// dx.doi.org/10.1007/s10072-014-1870-x

21. National Sleep Foundation. Depression and sleep [Internet]. Washington, DC: National Sleep Foundation; [cited Jul 1]. Available from: https://www.sleepfoundation.org/articles/ depression-and-sleep

22. Rochon PA. Drug prescribing for older adults. Toronto: UpToDate; 2019.

23. Alhola P, Polo-Kantola P. Sleep deprivation: Impact on cognitive performance. Neuropsychiatr Dis Treat. 2007 Oct;3(5):553-67.

24. Burke SL, Maramaldi P, Cadet T, Kukull W. Associations between depression, sleep disturbance and apolipoprotein E in the development of Alzheimer's disease: Dementia. Int Psychogeriatr. 2016 Sept;28(9):1409-24. http://dx.doi.org/10.1017/S1041610216000405

25. Xie L, Kang H, Xu Q, Chen MJ, Liao Y, Thiyagarajan M, et al. Sleep initiated fluid flux drives metabolite clearance from the adult brain. Science. 2013 Oct;342(6156):373-7. http://dx.doi.org/10.1126/ science. 1241224

26. Alzheimer's Society. Anxiety and dementia [Internet]. London: Alzheimer's Society; [cited Jul 1]. Available from https://www.alzheimers.org.uk/about-dementia/symptoms-and-diagnosis/anxiety

27. Johns Hopkins Medicine. Beyond memory loss: How to handle the other symptoms of Alzheimer's [Internet]. Baltimore: Johns Hopkins Medicine; [cited Jul 1]. Available from: https://www.hopkinsmedicine.org/health/conditions-and-diseases/alzheimers-disease/ beyond-memory-loss-how-to-handle-the-other-symptoms-of-alzheimers

28. Teri L, Ferretti LE, Gibbons LE, Logsdon RG, McCurry SM, Kukull WA, et al. Anxiety in Alzheimer's disease: Prevalence and comorbidity. J Geron Med Sci. 1999 Aug;54(7):M348-52. http://dx.doi. org/10.1093/gerona/54.7.M348

29. Becker E, Lorena Orellana Rios C, Lahmann C, Rucker G, Bauer J, Boeker M. Anxiety as a risk factor of Alzheimer's disease and vascular dementia. Brit J Phys. 2018 Nov;213(5):654-60. http://dx.doi. org/10.1192/bjp.2018.173

30. Peters R, Peters J, Warner J, Beckett N, Bulpitt C. Alcohol, dementia and cognitive decline in the elderly: A systematic review. Age Ageing. 2008 May;37:505-12. http://dx.doi.org/10.1093/ageing/ afn095

31. Heirene R, John B, Roderique-Davies G. Identification and evaluation of neuropsychological tools used in the assessment of alcohol-related cognitive impairment: A systematic review. Front Psychol. 2018 Dec;9(2618):1-42. http://dx.doi.org/10.3389/fpsyg.2018.02618 